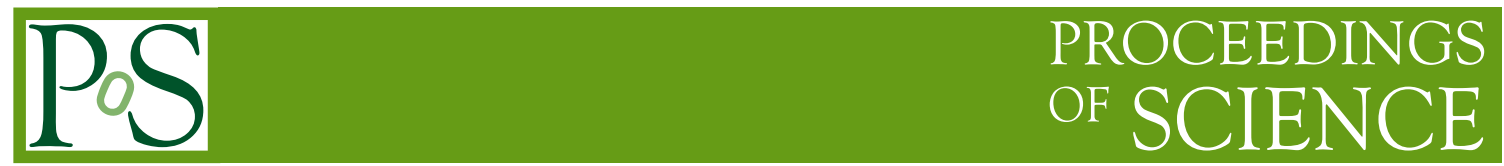

\title{
Irradiation and gas studies of Micromegas production chambers for the ATLAS New Small Wheel
}

\section{Lorenzo Pezzotti on behalf of the ATLAS Muon Collaboration}

Università degli Studi di Pavia \& INFN Sezione di Pavia

Via Agostino Bassi 6, Pavia, Italy

E-mail: lorenzo.pezzotti@cern.ch

One of the main upgrades of the ATLAS Experiment at the Large Hadron Collider (LHC) during Long Shutdown 2 is the replacement of the current forward muon spectrometer inner stations with a new detector, the New Small Wheel (NSW), covering the pseudorapidity region $1.3<\eta<2.7$. Large area micromegas (micro mesh gaseous structure) detectors will be installed as part of the upgrade in order to cope with the high radiation environment expected from the Run 3 onwards while providing high resolution tracking capabilities. Such large micromegas detector size (up to $3 \mathrm{~m}^{2}$ ) will be employed for the first time in high energy physics experiments, thus posing new challenges in terms of high-voltage and current stability. Before the installation within the ATLAS Detector, micromegas chambers are tested under irradiation at the CERN Gamma Irradiation Facility (GIF++).

40th International Conference on High Energy physics - ICHEP2020

July 28 - August 6, 2020

Prague, Czech Republic (virtual meeting) 


\section{The ATLAS New Small Wheel Project}

The New Small Wheel project [1] refers to the construction and installation of the two new innermost stations of the ATLAS [2] forward muon spectrometer with the final integration scheduled for the end of the Long Shutdown 2 (LS2). The project is dictated by the need to adopt tracking and triggering detectors with stable performance during the Large Hadron Collider High-Luminosity operation (HL-LHC) for which a luminosity up to $7.5 \times 10^{34} \mathrm{~cm}^{-2} \mathrm{~s}^{-1}$ is expected. The NSW will operate in the ATLAS pseudorapitidy region $1.3<\eta<2.7$ under high rates (up to $15 \mathrm{kHz} / \mathrm{cm}^{2}$ ) while reconstructing tracks with high precision and providing additional information to the original muon trigger system. The whole project is part of the ATLAS Phase-I upgrade focusing on the Level-1 trigger. The goal is to maintain the low-transverse momentum $\left(p_{\mathrm{T}}\right)$ trigger threshold for single leptons ( $e$ and $\mu$ ) while keeping the Level-1 rate at a manageable level.

The NSW layout adopts two detector technologies for planar gaseous detectors: the small-strip Thin Gap Chambers (sTGC), mainly devoted to triggering purposes given their single bunch crossing identification capability, and the micromegas (MM) detectors [3], providing highly precise hit information given their small gas gap and strip pitch. Each NSW consists of 16 multi-layer sectors. Each sector is made of six sTGC and four MM detectors arranged in order to maximize the distance between sTGCs (sTGC-MM-MM-sTGC). Each MM chamber provides four hits so that a muon passing through the NSW produces eight tracking hits. Sectors are divided into 8 small sectors and 8 large sectors. The NSW diameter is about $9 \mathrm{~m}$. Figure 1 shows the structure of the NSW (left) and the detectors composition for a single sector (right).

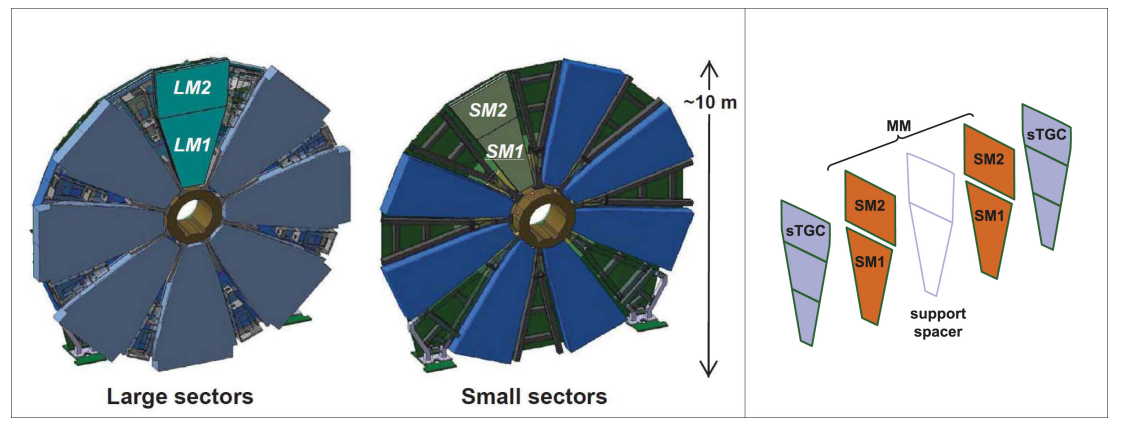

Figure 1: Structure of the NSW with sectors (left). Sketch of a single sector made of four MM and six sTGC chambers (right). Image from [4].

\section{Micromegas chambers}

A single layer of a micromegas detector is sketched in Figure 2 (left). The readout PCB, held at $500-600 \mathrm{~V}$ depending on the gas mixture, is followed by a grounded stainless steel mesh to define the $128 \mu \mathrm{m}$ wide avalanche region. The mesh is followed by a $5 \mathrm{~mm}$ drift gap ending with a cathode plane held at $-300 \mathrm{~V}$. The drifting electric field is $600 \mathrm{~V} / \mathrm{cm}$ and the typical avalanche electric field is $40-50 \mathrm{kV} / \mathrm{cm}$. The standard gas mixture is $\mathrm{ArCO}_{2}$ (93:7). The mesh, designed to be transparent to drifting electrons, is stretched over the readout PCB area and supported by $128 \mu \mathrm{m}$ high pillars glued on the PCB surface. The readout PCB consists of resistive strips deposited on 
a kapton ${ }^{\circledR}$ layer and the actual signal is carried by capacitively coupled copper strips below the insulator. Copper strips are printed on a $500 \mu \mathrm{m}$ thick FR4 layer. They are $17 \mu \mathrm{m}$ thick and $300 \mu \mathrm{m}$ wide. To increase the hit precise coordinate resolution the strip pitch was reduced down to $425 \mu \mathrm{m}$. Resistive strips are $300 \mu \mathrm{m}$ wide and their strip pitch is $425 \mu \mathrm{m}$. The design resistive strip resistivity is $\simeq 10 \mathrm{M} \Omega / \mathrm{cm}$.

Charged particles traversing the drift region ionize the gas and the electrons liberated drift towards the mesh. The charge amplification happens in the avalanche region. The electron drift is a slow process that takes tens of nanoseconds. The amplification step instead happens in a fraction of nanosecond inducing a fast pulse on the readout strip. Most of the ions are produced in the last avalanche steps, close to the readout strips, and their evacuation takes $\simeq 100 \mathrm{~ns}$, still very fast compared to other detectors. This fast positive charge evacuation makes MM chambers suited for high rate operations.

To achieve four tracking hits per MM chamber four readout planes per each detector are needed. The detector configuration chosen is shown in Figure 2 (right), called a quadruplet. Each quadruplet is made of five panels, two readout panels and three drift panels. The NSW will integrate four types of quadruplets called Small-Modules-1 (SM1) and Small-Modules-2 (SM2), to be used in small sectors, and Large-Modules-1 (LM1) and Large-Modules-2 (LM2) for the large sectors. Quadruplets are trapezoidal in shape. The SM1 and SM2 quadruplet surface is $\simeq 2 \mathrm{~m}^{2}$ while it reaches $\simeq 3 \mathrm{~m}^{2}$ for LM1 and LM2 quadruplets. Overall, NSW micromegas chambers will cover an active area of $\simeq 1280 \mathrm{~m}^{2}$. Each layer of SM1 and LM1 type chambers is divided in 10 independently powered sectors so that the whole detector is driven by 40 high-voltage (HV) lines. SM2 and LM2 chambers have 6 independently powered sectors per layer for a total of 24 high-voltage lines.

SM1, SM2, LM1 and LM2 detectors are built in Italy, Germany, France and Greece/Russia, respectively. Micromegas chambers are shipped to CERN from each site for final testing and integration. The high-voltage testing is performed under irradiation at the CERN Gamma Irradiation Facility (GIF++).

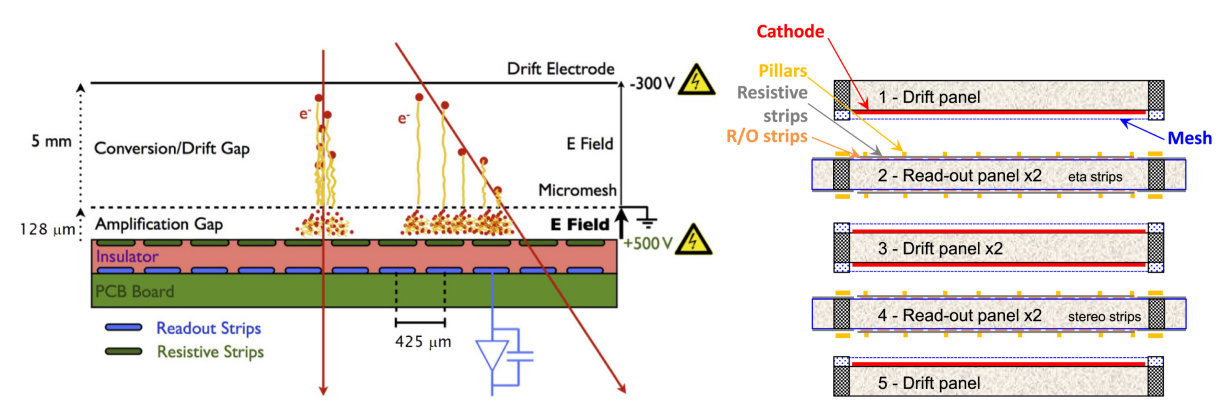

Figure 2: Scheme of a single layer MM chamber (left) and 4-readout layers configuration of the ATLAS MM chambers (right). Image from [4].

\section{Micromegas chambers high-voltage testing}

The Gamma Irradiation Facility (GIF++) [5], built during the Long Shutdown 1 at the CERN SPS North Area, is mainly used for the characterization of large-area gaseous detectors. It exploits 
a single $\simeq 14 \mathrm{TBq}{ }^{137} \mathrm{Cs}$ radioactive source with a half-time of 30.08 years. ${ }^{137} \mathrm{Cs}$ decays by beta emission to a metastable nuclear isomer of barium, ${ }^{137 m} \mathrm{Ba}$, with a branching ratio of $94.6 \%$. The remainder directly populates the ground state of ${ }^{137} \mathrm{Ba}$, which is stable. Metastable barium has a half-life of about $153 \mathrm{~s}$, and is responsible for all of the gamma ray emissions in samples of ${ }^{137} \mathrm{Cs}$, it decays to the ground state by emission of $662 \mathrm{keV}$ photons. The photon field is tunable with a set of lead filters.

Typically, micromegas chambers are positioned between $1 \mathrm{~m}$ and $3 \mathrm{~m}$ distances from the source, where a photon flux between $10^{6} \mathrm{~cm}^{-2} \mathrm{~s}^{-1}$ and $5 \times 10^{7} \mathrm{~cm}^{-2} \mathrm{~s}^{-1}$ is expected, according to Ref. [5]. The goal is to measure the current produced at the amplification stage with respect to the high-voltage applied and the incident flux. Particular attention is given to the current (in)stabilities problems and the spiking effects that might compromise the detector performance during the long-term operation within the ATLAS Detector. According to our measurements at a distance of $2 \mathrm{~m}$ from the source, taking into account the micromegas sensitivity to photons, a hit rate of $\simeq 40 \mathrm{kHz} / \mathrm{cm}^{2}$ is expected when no filter is inserted, more than 2 times higher than the one foreseen at the HL-LHC operation. The first micromegas chambers tested showed severe spiking issues. An example from a smallmodule- 2 type chamber, SM2 M12, is showed in Figure 3 (left). The current at the amplification stage (red line) is plotted when the detector is exposed to different fluxes by changing the GIF++ source filters. The nominal attenuation value is plotted as well (blue line). The current is completely dominated by spikes and streams originating at the amplification stage.

The only solution for problematic sectors is to lower the HV leading to a lower single-hit efficiency. A MM chamber is accepted for integration if its average efficiency is above $90 \%$ and the spike rate is less than six per minute: this condition occurs at an average $\mathrm{HV}$ of $560 \mathrm{~V}$. The nominal working point with the standard gas mixture is $570 \mathrm{~V}$. In the first months of 2019 about 7\%, 15\%, 30\% and $12 \%$ of the sectors were found below this specification respectively for SM1, SM2, LM1 and LM2 chambers. The detector production was delayed and eventually stopped for all construction sites while trying to solve the spiking problem. Several features were considered at the origin of the spiking behavior among which there were the humidity correlation, the non-perfect mesh stretching and the ionic contamination. The first hint of the real cause came after a small-module-1 type chamber, SM1 M3, was irradiated for three months at GIF++. The chamber was then shipped back to the assembly workshop at LNF (Laboratori Nazionali di Frascati) and re-opened. Strong signs of damaging of the readout PCBs were found close to the HV distribution line and the resistive strips interconnections closer to the HV distribution line. This triggered the idea that spikes occur where the local resistance of the resistive layout is below a certain threshold. If the resistance is too low the spikes quenching mechanism does not work anymore and the only way to lower the spike intensity is to lower the HV.

A technique to mitigate the problem was developed at LNF and later adopted by every construction site. It is known as passivation. The resistance of the resistive strips is measured, using a $1 \times 1 \mathrm{~cm}^{2}$ probe, per each MM sector at several points close to the HV distribution line. If the resistance is found to be lower than $0.8 \mathrm{M} \Omega$, an epoxy glue film is deposited over that region to electrically insulate the area. The epoxy film is typically few centimeters wide and reduces the detector active area. For instance, for SM1 type chambers, a $1 \mathrm{~cm}$ wide passivation would reduce the active area of the smallest PCB of $5 \%$ and the one of the largest PCB of $2 \%$. The technique helped in mitigating the spiking problems, sometimes with dramatic effects, for instance Figure 3 (right) shows the 
current at the amplification stage for the same sector after the passivation technique is applied.

Starting from the second half of 2019 all problematic chambers were treated with the passivation technique. The fraction of good sectors tested at CERN reached $98 \%$ for SM1 and SM2 type chambers, $90 \%$ for LM1 type chambers and $92 \%$ for LM2 type chambers.
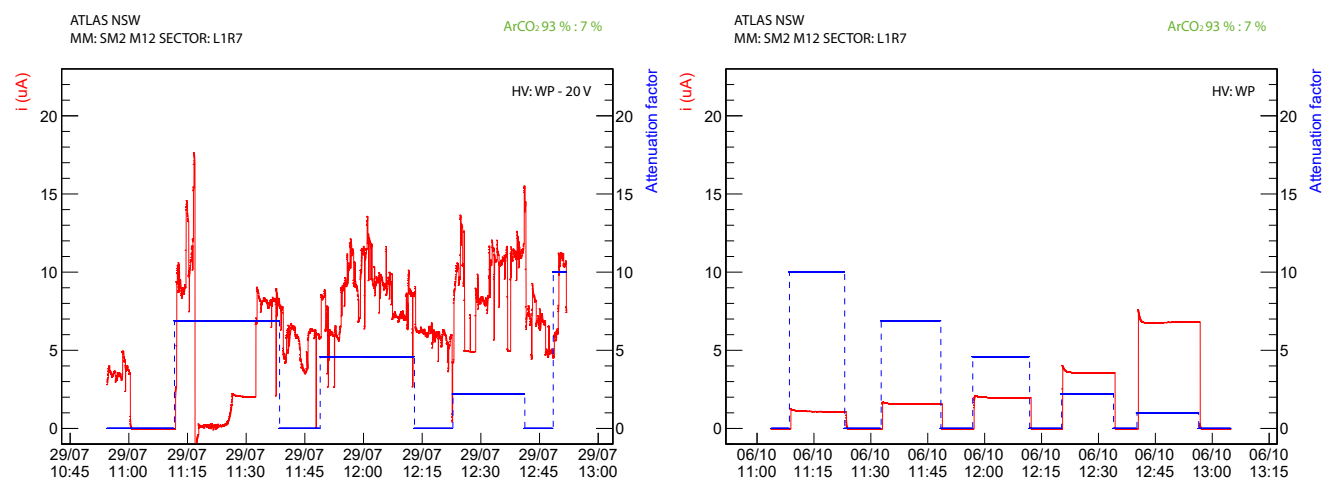

Figure 3: Monitored current at the amplification stage of chamber SM2 M12, sector L1R7, irradiated at GIF++ before (left) and after (right) passivation.

To further mitigate the micromegas chambers HV instabilities, the ATLAS Muon Collaboration started investigating the chambers $\mathrm{HV}$ behavior while using different gas mixtures. Three gas mixtures were considered: $\mathrm{ArCO}_{2}$ (93:7), which is the baseline one, $\mathrm{ArCO}_{2}$ (80:20) and $\mathrm{ArCO}_{2} \mathrm{C}_{4} \mathrm{H}_{10}$ (93:5:2). To compare the HV behaviour for the three gas mixtures, the current at different amplification voltages and particle rates was recorded. Results for chamber LM2 M7, sectors L1L6 and L3R7, are shown in Figure 4. The three gas mixtures define three different high-voltage operation ranges. The current values vs. the amplification voltage is well described by an exponential fit to data up to a certain current value. To highlight this point, an exponential fit to data was performed up to $20 \mathrm{~V}$ below the working point (colored lines) and the functions found were extrapolated till the maximum voltage values. In the very high current regions all current values lie below the exponential prediction of the current value. This effect is caused by a voltage drop at the amplification stage when very high currents are produced.

Polyatomic gases, like isobutane $\left(\mathrm{C}_{4} \mathrm{H}_{10}\right)$, act as quenchers by absorbing the radiated photons and dissipating their energy through dissociation or elastic collisions. Indeed, we found dramatic improvements in spike suppression when flushing the $\mathrm{ArCO}_{2} \mathrm{C}_{4} \mathrm{H}_{10}$ (93:5:2) mixture. This is also true for passivated chambers that still show spiking problems, for instance Figure 5 shows the monitored current of chamber SM1 M31, sector L3R5, when exposed to different photon fluxes (blue line corresponding to the GIF++ field nominal attenuation). The spiking and streaming issues found with $\mathrm{ArCO}_{2}$ (93:7) (left) completely disappear by using the isobutane enriched mixture (right).

\section{Conclusions}

The New Small Wheel upgrade of the ATLAS Experiment at the LHC is proceeding, with the NSW-A completed and NSW-C under construction. The main source of micromegas chambers high-voltage instabilities has been linked to the low resistivity of the resistive strips layout thanks 

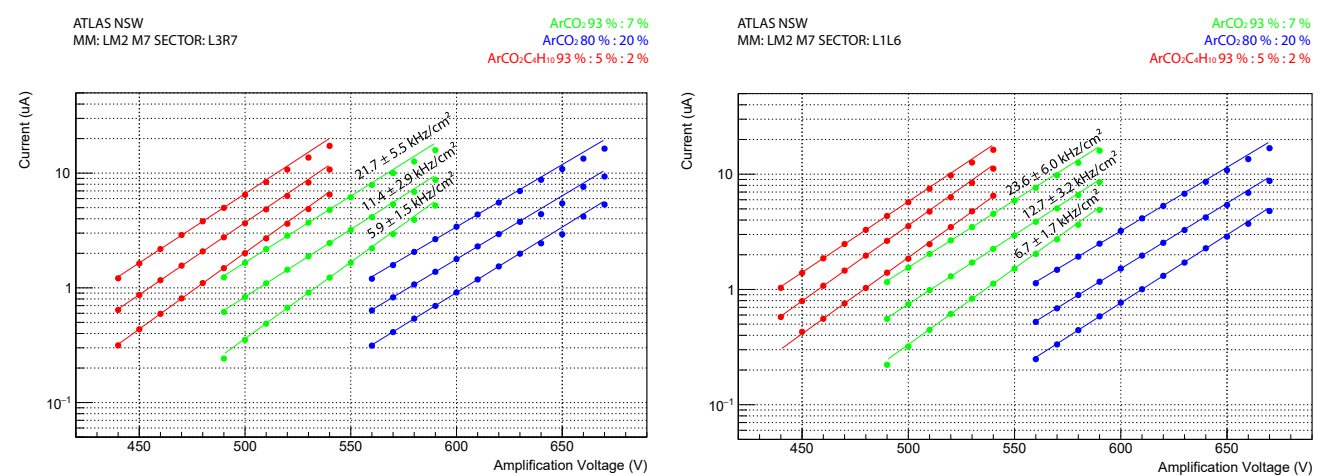

Figure 4: Current produced at the amplification stage versus the amplification voltage for different gas mixtures and particle rates. Results for chamber LM2 M7, sector L3R7 (left) and L1L6 (right).
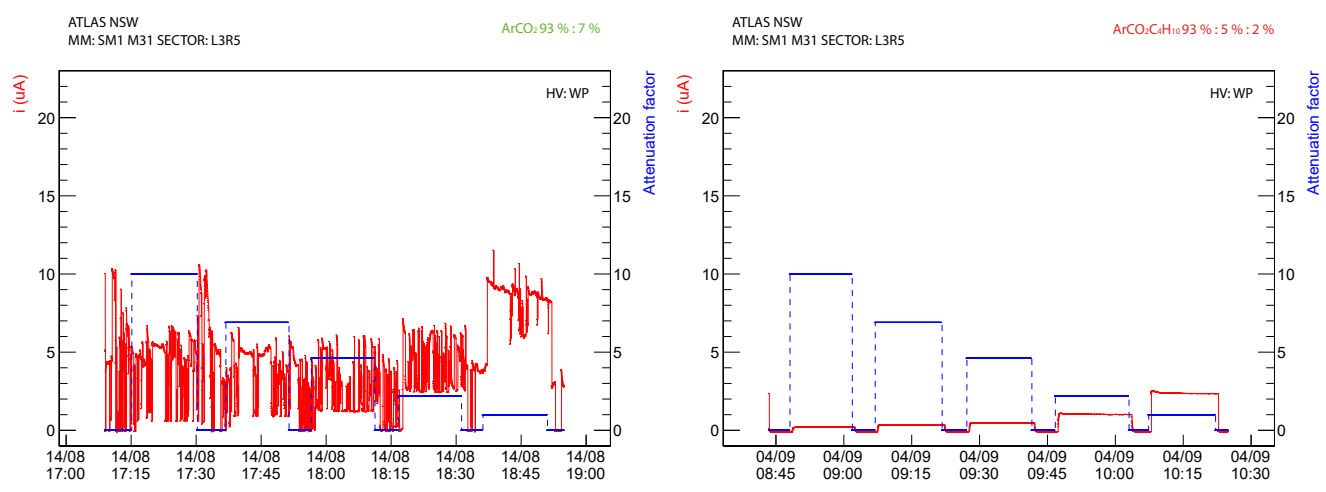

Figure 5: Monitored current at the amplification stage of sector L3R5, chamber SM1 M31 (passivated), when the chamber is operated at the working point and is exposed to different hit rates. Left side corresponds to the chamber flushed with $\mathrm{ArCO}_{2}$ (93:7), right side corresponds to the chamber flushed with $\mathrm{ArCO}_{2} \mathrm{C}_{4} \mathrm{H}_{10}$ (93:5:2).

to chambers high-voltage testing at the CERN Gamma Irradiation Facility. An ad hoc solution was found and the overall quality of the detectors improved. Currently gas studies exploiting spike quenchers are ongoing with interesting results.

\section{References}

[1] ATLAS Collaboration, CERN-LHCC-2013-006, ATLAS-TDR-20-2013.

[2] ATLAS Collaboration, JINST 3 S08003.

[3] Alexopoulos T. et al., Nucl. Instr. and Meth. A 640 (2011) 110-118.

[4] Alexopoulos T. et al., Nucl. Instr. and Meth. A 955 (2020) 162086.

[5] Pfeiffer D. et al., Nucl. Instr. and Meth. A 866 (2017) 91-103. 